\title{
L'exploitation des ressources thermales à Dax
}

\author{
par Pascal Counilh, Jean-Luc Gibert, Nancy Lopez \\ de la Régie Municipale des Eaux de la Ville de Dax
}

\section{INTRODUCTION}

Le thermalisme utilise les sources d'eau minérale naturelle pour leurs propriétés thérapeutiques au sein des stations thermales. En France, 650000 curistes sont ainsi traités chaque année. Plus de 1000 sources sont inventoriées dans 104 stations thermales.

Dax revendique l'utilisation thermale de ses eaux depuis l'époque romaine. Mais le thermalisme moderne à Dax doit son développement à l'accès de tous à cette thérapie grâce au remboursement des soins thermaux par l'assurance-maladie.

Dax, première station thermale de France, est la station du rhumatisme. On y trouve toutes les techniques d'hydrothérapie, mais la spécificité de Dax est d'avoir développé une technique particulière, à base d'une boue thermale originale «le Péloïde de Dax »: la pélothérapie. La boue est le résultat du séjour prolongé d'un substrat minéral au contact de l'eau chaude, favorisant le développement d'une biocénose spécifique.

L'eau minérale est donc un élément déterminant pour le thermalisme dacquois. Sa production, sa distribution et le contrôle de sa qualité font l'objet d'une gestion rigoureuse et attentive de la part de la Régie des Eaux de Dax, service municipal chargé de ces missions.

\section{Q QUELQUES DÉFINITIONS}

Le thermalisme est l'ensemble des moyens administratifs, médicaux, hospitaliers, sociaux et hôteliers mis en œuvre pour l'utilisation thérapeutique des eaux de sources, qu'elles soient chaudes ou non.

La crénothérapie concerne les pratiques thermales. Elle fait appel aux eaux naturelles contenant en solution plusieurs sels minéraux, et à leurs dérivés (gaz, boues). La minéralisation, la température et le flux de ce type d'eaux doivent rester stables et préservés de toutes pollutions au point d'émergence.
Une eau minérale naturelle est une eau possédant un ensemble de caractéristiques qui sont de nature à lui apporter des propriétés favorables à la santé. Elle se distingue nettement des eaux destinées à la consommation humaine:

- par sa nature caractérisée par sa teneur en minéraux, oligo-éléments ou autres constituants et par certains effets,

- par sa pureté originelle.

L'une et l'autre de ces caractéristiques sont conservées intactes en raison de l'origine souterraine de cette eau à l'abri de tout risque de pollution.

Elle provient d'une nappe ou d'un gisement souterrain exploité à partir d'une ou plusieurs émergences naturelles ou forées.

Elle témoigne dans le cadre des fluctuations naturelles connues d'une stabilité de ses caractéristiques essentielles notamment de composition et de température à l'émergence, qui n'est pas affectée par le débit de l'eau prélevé.

\section{III - ORIGINE DE L'EAU MINÉRALE DE DAX}

L'origine des eaux minérales de Dax est sans nul doute météorique. Des eaux de pluies s'infiltrent, profitant de terrains perméables, sur la bordure Est du Bassin Aquitain. En gagnant la profondeur, ces eaux s'enrichissent en sels minéraux et augmentent leur température au contact de la roche. Ces eaux chaudes vont cheminer sur de longues distances pendant plus de 3000 ans pour parvenir dans la basse vallée de l'Adour, où se situe Dax. Cette région est caractérisée par une structure géologique complexe, qui a favorisé la remontée de nombreux massifs de sels (diapirisme) débouchant ou non à la surface. Ces structures ont plissé et redressé les calcaires et dolomies qui contiennent la nappe d'eau chaude et permis l'instauration de circulations ascendantes. Elles se manifestent par les sources d'eaux thermales dont certaines sont captées depuis l'époque gallo-romaine.

Au cours de la remontée des eaux, le drain thermal princi-

The DAX water local administration provides the hydropathic establishments of the spa with mineral water supply. The today's warm water production which amounts to more than $7,500,000 \mathrm{~m}^{3}$ is one of the highest production of natural warm thermal water in France and makes of DAX the first French spa that is visited by 55,000 people per year. 
pal de la roche dolomitique qui constitue le réservoir, tend à se ramifier en plusieurs branches donnant lieu à l'ensemble des sources de Dax.

Le pôle principal se situe autour de la Fontaine-Chaude où l'on rencontre la température, la minéralisation et le débit les plus élevés.

\section{RARACTÉRISTIQUES PHYSICO- CHIMIQUES DE L'EAU MINÉRALE DE DAX}

L'eau thermale de Dax est une eau sulfatée calcique légèrement chlorurée sodique. Elle est peu minéralisée (extrait sec 1 gramme par litre) mais comporte de nombreux oligo-éléments dont certains comme le strontium et le fluor à des teneurs importantes de l'ordre du mg/l. Les eaux de Dax, comme c'est le cas des eaux d'origine profonde, sont très légèrement radioactives. La circulation profonde et la température élevée à l'émergence confèrent à l'eau une pureté microbiologique de grande qualité.

\section{LA PRODUCTION DE L'EAU MINÉ- RALE DE DAX}

La production est donc assurée par un service municipal. Elle s'élève aujourd'hui à plus de $1500000 \mathrm{~m}^{3}$ par an, ce qui permet de mettre à la disposition de chaque curiste un volume très important d'eau chaude, environ $22 \mathrm{~m}^{3}$.
La station de Dax connue pour ces eaux chaudes n'a plus de nos jours de sources naturelles. Toute la production est assurée par des pompages. Aujourd'hui, cing forages thermaux exploitent la nappe d'eau chaude. Tous situés sur la rive gauche de l'Adour, leur profondeur varie entre $56 \mathrm{et}$ 538 mètres et la température de l'eau de 54 à $62,5^{\circ} \mathrm{C}$.

\section{VI 圈 LA DISTRIBUTION DE L'EAU MINÉ- RALE DE DAX}

La distribution de l'eau aux 16 établissements thermaux et 2 centres de réducation fonctionnelle de la station se fait par un réseau, constitué d'une boucle de 6 kilomètres de canalisations en matériau composite (résine époxy-fïbre de verre), isolées par la mousse de polyuréthanne. Les performances mécaniques, thermiques et hydrauliques de ce réseau permettent à l'eau de conserver ses caractéristiques originelles.

Les centres de soin sont raccordés au réseau par l'intermédiaire d'un branchement unique qui comporte des organes de contrôle et de régulation calibrés selon la taille de l'établissement desservi.

\section{VII 圆 LE CONTRÔLE DE QUALITÉ}

Pour favoriser une bonne pratique thermale et, garantir aux établissements thermaux desservis, une thérapeutique thermale de qualité, la Ville de Dax a mis en place un système d'assurance-qualité des produits qu'elle exploite (eau minérale) et qu'elle prépare (boue thermale).
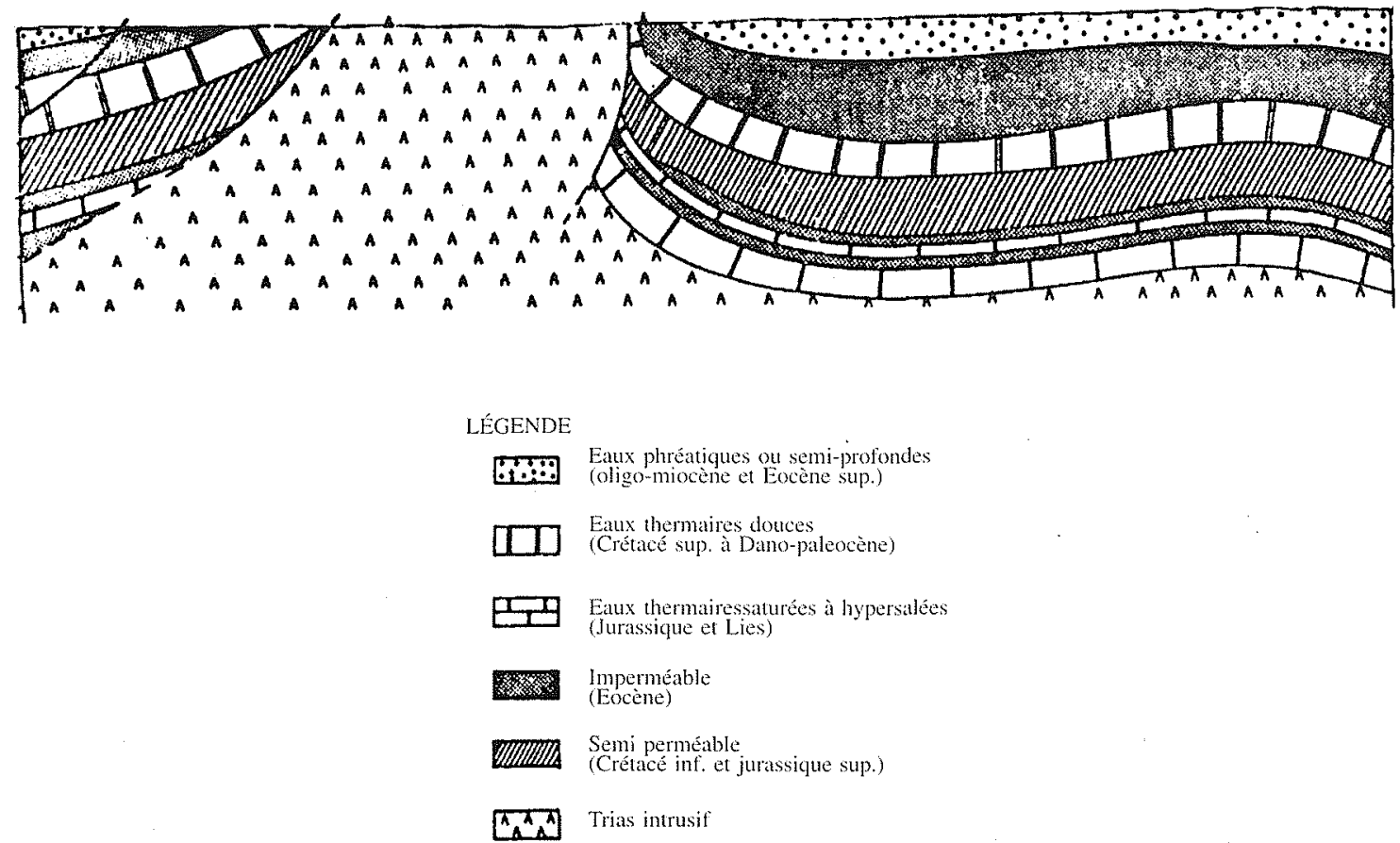

1. Coupe hydro-lithologique dans le Bas-Adour: 
Compte tenu du caractère géologique particulier du soussol dacquois et de l'interférence de la nappe thermale avec les aquifères froids superficiels, une gestion et un contrôle rigoureux de la ressource sont nécessaires. Cette gestion et ce contrôle sont exercés à la production, sur le réseau, sur les ouvrages de production, dans le choix des matériaux et enfin, dans les établissements thermaux.

\subsection{Contrôle de l'eau minérale à la production}

Tous les forages sont dotés de chaîne de mesure et d'enregistrement qui procurent une surveillance quotidienne des pressions, niveaux, températures et débits de l'eaut. Un équipement de capteurs spécifiques permet également la détermination de la résistivité et, sur quelques points, de la turbidité.

A partir de ces relevés, les données sont archivées sur informatique (logiciel CHRONO du BRGM) pour analyser dans le temps les éventuelles variations de ces paramètres. Il est ainsi aisé de vérifier si les caractéristiques des forages sont normales par rapport aux situations observées à l'origine. A partir de là, des corrections notamment de débit peuvent être apportées sur les ouvrages de production. Ces décisions sont facilitées par l'utilisation d'un logiciel spécifique ADAGES, développé par la Régie des Eaux et le BRGM, qui offre le choix des débits d'exploitation des forages thermaux en fonction de la satisfaction d'une contrainte particulière (température, rabattement de la nappe thermale, teneur en sulfates de l'eau).

La surveillance de ces paramètres physico-chimiques est complétée par des analyses bactériologiques qui à l'encontre d'un problème particulier (une chute de température par exemple) donnent des précisions sur l'origine du désordre (infiltration d'eaux contaminées de surface par exemple) et guident utilement l'exploitant dans le choix de mesures conservatoires à prendre.

Nous avons pu mesurer l'intérêt de la méthode lors d'un incident constaté sur un forage situé en zone inondable dont la tête présentait un léger défaut d'étanchéité.

Les déterminations bactériologiques correspondent à celles préconisées par la réglementation sur les eaux minérales du 27 juillet 1992, y compris la recherche des légionelles.

Le traitement des domées ainsi recueillies, tant physiques que physico-chimiques, offre la possibilité d'étudier par forage les relations entre certains paramètres telles que «température-résistivité » ou «résistivité-niveau dynamique ». Ce type de relation montre la caractéristique fondamentale d'un forage thermal, c'est-à-dire le couple de paramètres «température-résistivité » et son évolution en fonction du niveau de l'eau.

L'examen régulier des valeurs de ces paramètres favorise une exploitation raisonnable des forages les plus vulnérables et permet d'éviter les surexploitations.

Ce système de surveillance de la ressource thermale constitue outre sa fonction d'analyse un outil de prévision à court et moyen terme.

\subsection{Contrôle de l'eau minérale sur le réseau}

Tous les établissements thermaux sont raccordés sur le réseau par un branchement unique. Le branchement est équipé, en plus des appareils de comptage, de tous les organes pour une bonne régulation du débit et notamment, un hydrolimiteur réduit les brusques élévations de consommation. Chaque fosse de raccordement est équipée d'un robinet de prélèvement qui permet le contrôle de la qualité de l'eau à l'entrée de l'établissement.

Les déterminations portent essentiellement sur la microbiologie de l'eau. Les conduites constituant le réseau ont été choisies après des tests de contact à l'eau thermale et, des analyses réalisées par le Centre de Recherche de la Ville de Paris. Ces tests ont permis de retenir le matériau le plus inerte vis-à-vis de l'eau minérale, la surveillance de la qualité de l'eau peut s'alléger de contrôles réguliers des paramètres physico-chimiques. En règle générale, les caractéristiques du point d'émergence sont conservées.

Il faut ajouter qu'à l'intersaison le réseau de distribution de l'eau minérale est entièrement nettoyé par l'introduction dans les conduites de pistons racleurs qui détachent les dépôts éventuels fixés sur les tubes. Cette opération de nettoyage, qui dure 4 semaines, se termine par une désinfection générale du réseau, par l'injection d'eau de Javel. Un rinçage très abondant précède la remise en eau chaude du réseau.

Enfin, il faut préciser que le réseau est protégé d’éventuels retours d'eau de la part des établissements thermaux par une disconnexion complete du raccordement. La délivrance se fait toujours par surverse dans une bâche.

\subsection{Contrôle des ouvrages de production}

Si l'examen de la qualité de l'eau permet également dans certains cas d'effectuer un diagnostic sur l'état de l'ouvrage de production, celui-ci est généralement insuffisant pour guider l'exploitant dans la prévention de désordres qui affectent le forage.

La Régie des Eaux procède systématiquement à des contrôles plus approfondis. Tous les cinq ans, est pratiqué un bilan complet de chaque forage par la mise en auvre d'une inspection vidéo, complétée de diagraphies (thermographie, micro-moulinet) et de la détermination de l'hydraulicité de l'ouvrage et de l'aquifère (essai de pompage par paliers)

Cette surveillance facilite la mise en évidence des processus de dégradation des forages avant même qu'ils n'affectent la qualité de l'eau thermale comme par exemple la rupture d'un caising, les défauts d'une cimentation, le colmatage d'une crépine,...

On peut intervenir ainsi préventivement pour éviter l'altération toujours préjudiciable de la qualité de l'eau ou l'apparition de désordres beaucoup plus graves et souvent très coûteux à résorber.

\subsection{Contrôle des matériaux}

Des précautions doivent être prises quant au choix des matériaux en contact avec de l'eau thermale. Ils doivent altérer le moins possible la qualité de l'eau et résister à son agressivité.

Si les tests décrits plus haut ont permis de retenir des tuyaux en produits composites (résine, époxy fïbre de verre) pour les canalisations, d'autres essais ont sélectionné les types de revêtement à mettre sur les vannes ou la nature des joints utilisés.

Il faut aussi signaler que l'exploitation du réseau par les équipes d'intervention se fait suivant des protocoles bien établis. Ainsi par exemple, tout changement d'une pièce ou d'un appareil se fait après désinfection de l'élément de remplacement.

\subsection{Contrôle dans les établissements thermaux}

Soucieux de garantir une qualité irréprochable de leurs installations, les établissements thermaux de Dax ont contribué à créer et ont signé la Charte QUALITHERME. Grâce à 
cette charte de qualité le Laboratoire Municipal peut pénétrer dans les établissements thermaux pour effectuer des prélèvements inopinés sur les principaux points d'utilisation de l'eau thermale (buvette, piscine, baignoires,...). Ces contrôles volontaires se font en complément de la surveillance officielle exercée par l'administration. Selon les résultats des analyses, des programmes de nettoyage et de désinfection des installations sont mis en place si besoin est.

Tous les établissements ont également fait part de leurs plans d'hygiène aux services de la DDASS.

L'ensemble des contrôles ainsi réalisés permet d'une part, de délivrer aux établissements thermaux de soins une eau thermale aux caractéristiques constantes et, donc, de préserver ses propriétés thérapeutiques et, d'autre part de gérer au mieux la ressource thermale pour assurer sa pérennité. Il garantit enfin aux curistes la qualité du produit dans l'établissement thermal. Toutes les procédures et protocoles sont notés dans des cahiers destinés aux agents d'exploitation.

\section{CONCLUSION}

Quelques chiffres peuvent faire comprendre l'importance du thermalisme à Dax:

- $1^{\text {re }}$ station thermale de France avec 55000 curistes,

- 75 médecins thermaux

- 18 établissements thermaux.

- 5000 emplois

- un chiffre d'affaires de 500 millions de francs,

- 1725 chambres d'hôtels, 1272 studios hôteliers, 966 locations meublées, 311 places en camping,

- 400 commerces.

On peut concevoir ainsi que cette économie repose sur la pérennité d'une ressource en eau minérale sur le plan quantitatif et qualitatif.

Tous les efforts en matière de contrôle et d'assurance-qualité que la Ville de Dax a réalisés montrent qu'elle considère ses produits thermaux, eau minérale et Péloïde de Dax, comme des «médicaments thermaux », à la préparation et au contrôle desquels elle attache un soin et une rigueur scientifiques. 